\title{
VOCs vapour sensor based on polyaniline salts films and quartz crystal microbalance.
}

\author{
Ahmed Mekki ${ }^{1}$, Boualem Mettai ${ }^{1}$, Zaher Ihdene ${ }^{1}$, Rachid Mahmoud ${ }^{1}$, Zineb Mekhalif ${ }^{2}$ \\ ${ }^{1}$ Ecole Militaire Polytechnique EMP, BP 17, Bordj-El-Bahri, Alger, Algérie. \\ Corresponding author: Ahmed Mekki-Email address: mekki_ahmedkarim@yahoo.fr \\ ${ }^{2}$ Laboratoire de Chimie et d'Electrochimie des Surfaces, FUNDP, Rue de Bruxelles, 61, B-5000, \\ Namur, Belgique.
}

\begin{abstract}
A sensor based on the quartz crystal microbalance (QCM) technique was used for the detection of a number of organic volatile compounds (VOCs) vapours such as aromatics, alcohols and amphoteric solvents. Detection was mainly based on sensitive and thin films of synthesized polyaniline, emeraldine salt (ES), doped with three different dopants ( $\mathrm{HCl}$, DBSA and NDSA) prepared by dip coating of a QCM electrode. Values of the frequency shifts $(\Delta f)$ were found to be linearly correlated with the (VOCs) concentration given in part per million (ppm). The changes in the frequency are principally due to the establishment of electrostatic interaction developed between the ES films and sorbents as well as the sorbent type. The sensitivity of the sensor was found to be governed by the dopant type and the thickness of the prepared thin layer. The sensor has shown a good reproducibility and reversibility.
\end{abstract}

Key words: sensor, quartz crystal microbalance (QCM, polyaniline emeraldine salt (ES), dopants $(\mathrm{HCl}, \mathrm{DBSA}$ and NDSA), dip coating, electrostatic interaction.

\section{Introduction}

Owing to concerns about environmental protection, human health care and quality control, a great attention of various air pollution control agencies has been paid to the detection of toxic odorants and airborne volatile organic compounds (VOCs). Considerable quantities of VOCs are used in many workplaces, manufacturing units and facilities. A group of VOCs, including volatile organic solvents such as aromatics (benzene, toluene and xylene isomers) and alcohols, are widely used in laboratories, medicine and food industry. They create serious effects on the quality and consequently, human health by causing some nerve diseases and even death. Thus, the on line monitoring of these highly threatening (VOCs) in air is an imperative task. Therefore, several techniques and materials were used for their detection such as Fourier transform infrared spectrometry (FTIR), gas chromatography (GC), mass spectrometry (MS) and other methods based on metal oxides [13]. However, these techniques are not suitable for the on-line monitoring and they need expensive instrumentations and are timeconsuming. In the last few decades, efforts are focused on the development of sensors with more selectivity and reproducibility for on-line monitoring and in situ detection of VOCs.
Therefore, a number of chemical sensors and sensors array have been developed, mainly depending on the use of different sensitive coatings to a special analyte [4]. A great interest is devoted to the development of surface acoustic wave (SAW) and Quartz Crystal Microbalances (QCM) sensors for low cost, compact volume easy portability and high sensitivity. The use of a SAW sensor to detect chemical vapors was first reported in 1979 and has since been widely studied where the detection is based on the partition of the analytes between the environment surrounding the sensor and a sensitive layer. Meanwhile, QCM sensor has great interest in its lower operation temperature for detection of VOCs and is sensitive to mass change. The change in mass onto the quartz crystal electrode during the reaction with adsorbent was used as sensor for interested vapours and gases. The changes in masse $m^{\prime}$ of the sensitive layer can be measured by the oscillating frequency of the quartz electrode. The frequency change $\Delta f$ to the mass loaded is calculated from the Sauerbrey equation:

$\Delta f=-\left(\frac{2 f_{0}^{2}}{\sqrt{\rho_{Q} \mu_{Q}}}\right) m^{\prime}$

Where $f_{0}(\mathrm{~Hz})$ is the natural frequency of the quartz crystal, $\rho_{Q}$ is the quartz density $(2.649 \mathrm{~g}$. 
$\mathrm{cm}^{-3}$ ) and $\mu_{Q}$ the shear modulus $\left(2.947 \times 10^{10} \mathrm{~N} / \mathrm{m}^{2}\right)$.

Amongst the various tested polymers, the utilization of conducting polymers as sensitive layers in gas sensors is becoming a promising approach. Polypyrrole was one of the first polymers used in gas sensor for detection of alcohols and other organic vapors [5-7], however it shows a low sensitivity, and incomplete desorption of the gas molecules Nowadays, the researchers are extending their efforts toward polyaniline as gas sensing material, which has demonstrated a good gas sensing ability due to its environmental stability, high electrical conductivity, good reversibility and reproducibility and good performance at room temperature $[8,9]$.

Although the plausible development that gas sensors based on conducting polymers are knowing, the exact nature of deriving interactions between analyte molecule and conducting polymer surface remains largely unknown and no reports are available. Thus, interactions identification is essential for selection of adequate conducting polymer that will be applied in chemical sensor.

\section{Results}

\section{Preparation of PANIs}

All chemicals were used as received, aniline purchased from Panreac (purity $>99 \%$ ), reagents grade ammonium peroxydisulfate, ammonium hydroxide and hydrochloric acid, were obtained from Prolabo, dodecylbenzensulfonic acid and 1,5Naphthalenedisulfonic acid were supplied by Sigma-Aldrich with a mass fraction purity greater than $98 \%$. Deionized water was used throughout the experiment. Polyaniline complexes with the three acids were prepared and purified according to methods described in reference [10]. As the polymerization has proceeded, the PANI films were dip coated onto the electrode of the QCM at different periods of time ranging from 30 to 120 minutes. The ES films coated on the electrode of the QCM were rinsed with deionized water and then dried in the oven at $50-60^{\circ} \mathrm{C}$ at least $30 \mathrm{~min}$ until the QCM attains constant frequency, the thickness of the film, $L(\mathrm{~cm})$ can be determined from the density of the ES, $\rho=1.44 \mathrm{~g} / \mathrm{cm}^{3}$ using the relation: $m^{\prime}=\rho L$.

\section{Procedures}

All measurements were carried out in a polyethylene cell with an internal volume of 140 $\mathrm{ml}$ at room temperature $\left(25^{\circ} \mathrm{C}\right)$ using a 10 $\mathrm{MHz}$ At-cut quartz crystal with $\mathrm{Ag}$ electrodes of $4 \mathrm{~mm}$ diameter on both sides. The frequency was measured using a counter, model Hewlett Packard 5383 A. Hamilton microliter syringe was used for VOCs injection after their evaporation. The concentration of injected analyte in the cell was determined in part per million (ppm) according to the following equation:

$C=\frac{22.4 \rho T V_{s}}{273 M V} \times 10^{3}$

Where $C$ is the concentration given in ppm, $\rho$ is the density of liquid sample in $\mathrm{g} / \mathrm{mL}$, $T$ is the temperature of detection chamber in Kelvin, $\mathrm{V}_{\mathrm{s}}$ is the volume of liquid sample in $\mu \mathrm{L}, \mathrm{M}$ is the molecular weight of sample in $\mathrm{g}$, and $\mathrm{V}$ is the chamber volume in liter.

After have been exposed The (ES) films coating the QCM electrodes to a specific concentration of VOCs vapours and the frequency variation with time has attained the steady state, they were exposed to hot nitrogen to desorb vapours and recover the electrode. The frequency backshift of the crystal to its initial value was taken as an indication of full desorption.

The thin films of (ES) coated on the QCM electrode have shown a sensitive detection for the injected VOCs, the frequency of the quartz crystal decreased due to the adsorption of the vapours into the ES films, according to the equation (1). After few minutes the equilibrium has been reached, the relation between frequency shift, $(\Delta f)$, and time was plotted for the adsorption of $p$-xylene vapour on the three polymers and is given on figure 1. From the figure, it is observed that the Es films doped (DBSA) are more sensitive and have higher response in term of $(\Delta f)$ variation comparatively to the other (ES) tested films. This result is plainly attributed to the polar character of the polymer doped (DBSA) which promotes the establishment of strong interactions relatively to those developed with the other Es films, particularly with the hydrophilic character of the dopant which contribute to the hydrogen bond establishment and electrostatic interactions with tested molecules. The interaction between the ES films and p-xylene is electrostatic interaction through the hydrogen bonding between the hydrogen atom of $p$-xylene and oxygen atom of the DBSA dopant and also the dispersive interactions developed between the aromatic rings of both $p$-xylene and the DBSA dopant. 


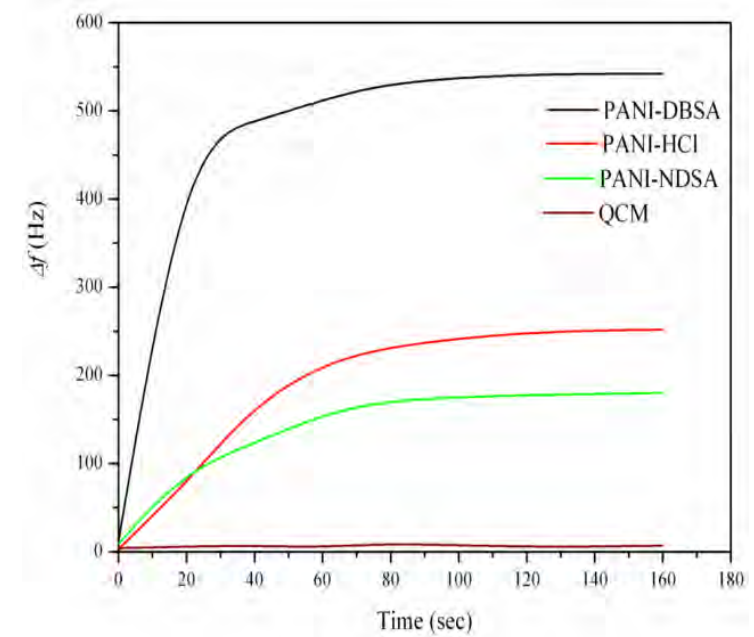

Fig 1: Frequency shift of emeradine salt (ES) coated on QCM electrode when exposed to the same concentration of $p$-xylene vapour.

It is also expectable that the polymer thickness would affect the response time and the sensitivity of the QCM sensor. The frequency of the QCM electrode having the different Es film thickness was recorded upon exposure to various concentrations of injected aromatics; it is worth noticeable that it was difficult to produce all the ES films with the same thickness; owing to the difficulty of controlling the deposition operation applied by the dipcoating method. Plots of $(\Delta f)$ with the concentration of $p$-xylene for different ES films of different thicknesses are shown in fig.2-4. We can observe that as the film thickness increases, the magnitude of $(\Delta f)$ and consequently the slope of correlation also increases. this is mainly due to the increasing of the active sites in the polymer and the polarity strength derived from the dopant presence.

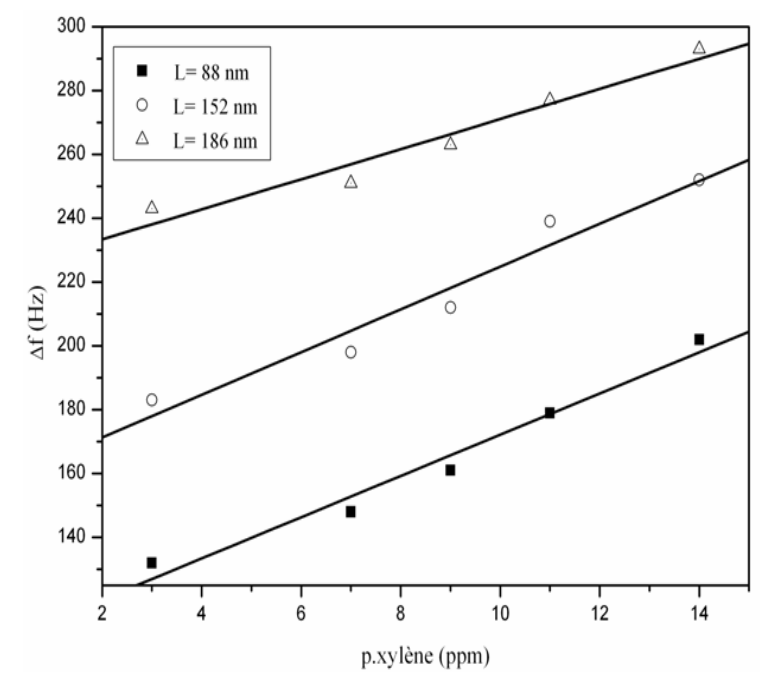

Fig 2: The effect of film PANI-HCl thickness on the detection of $p$-xylene vapours.

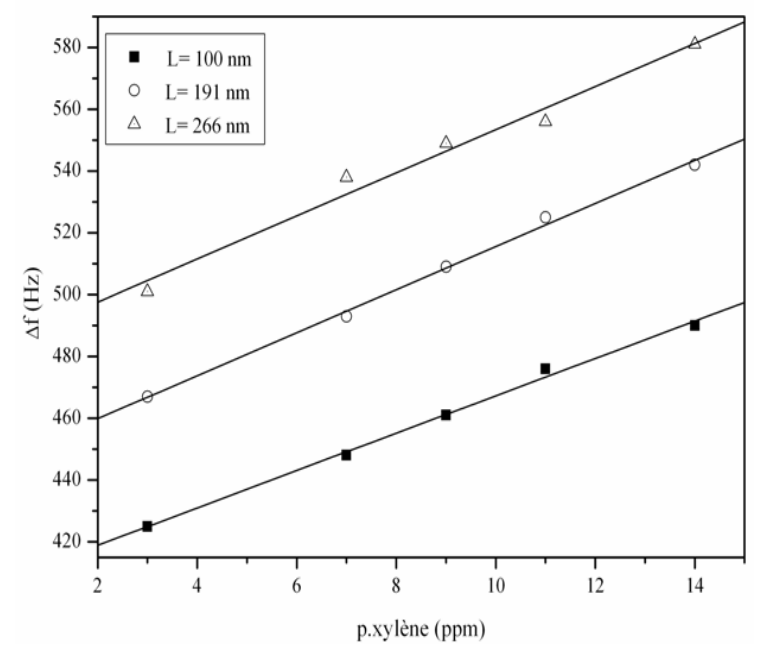

Fig 3: The effect of film PANI-DBSA thickness on the detection of $p$-xylene vapours.

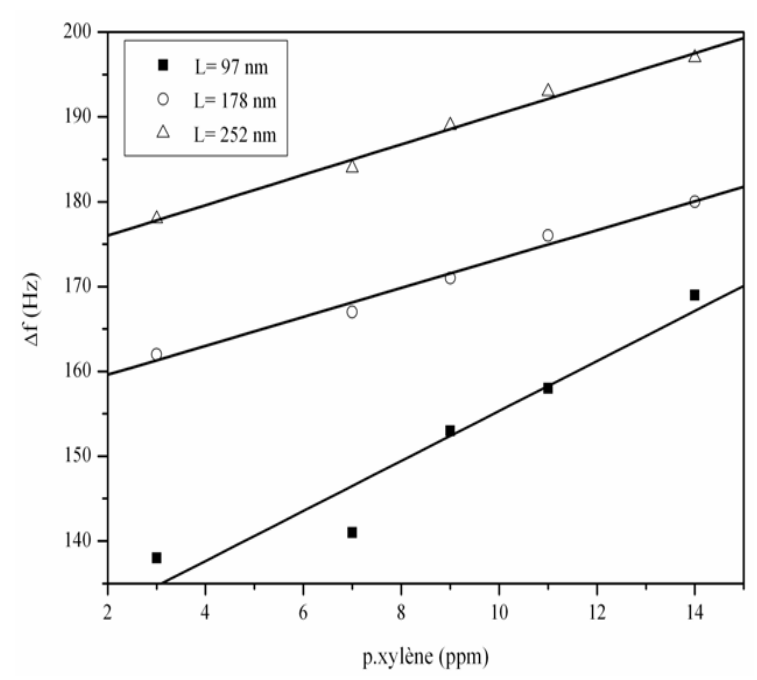

Fig 4: The effect of film PANI-NDSA thickness on the detection of $p$-xylene vapours.

\section{Reproducibility and reversibility of the sensor}

The sensor has shown a good reproducibility and reversibility. Figure 5 shows the exposure of the PANI-DBSA film having the thickness of $191 \mathrm{~nm}$ to the concentration of aromatics vapours included between 14 and $20 \mathrm{ppm}$ during two cycles (injection-desorption) on a period of 160 seconds, wherein $(\Delta f)$ was recorded versus time till reached the steady state and then the desorption of the crystal was obtained by using the hot dry nitrogen. The experiment was repeated for five times to insure complete reproducibility and reversibility of the sensor. 


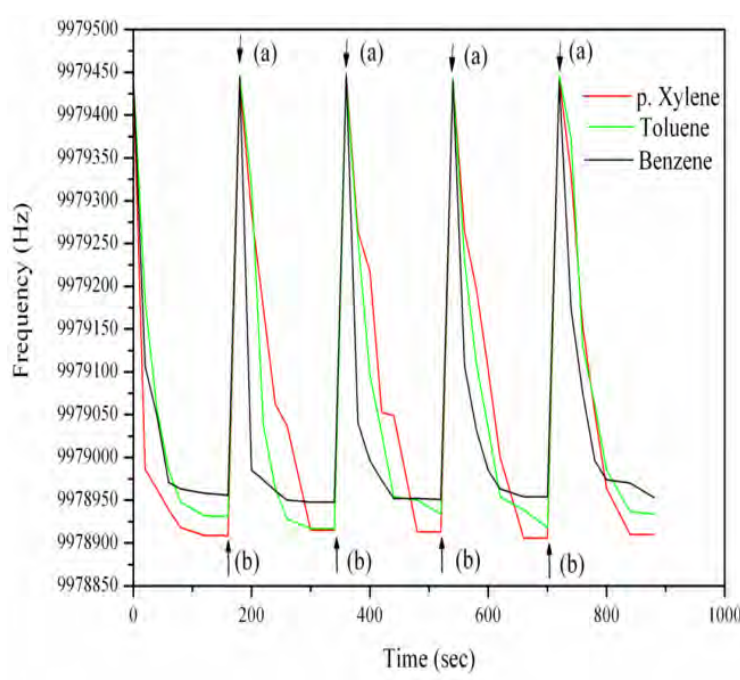

Fig 5: Reproducibility and reversibility of the QCM electrode coated with the PANIDBSA film when exposed to aromatics vapours: (a) injection, (b) desorption using hot dry nitrogen.

\section{Conclusion}

PANI films in the form of the ES dip-coated on the QCM electrode sensor have shown a good response, sensitivity and reproducibility with the tested VOCs trough the electrostatic interaction, particularly the Hydrogen and dispersive bonding. It was found that the dopant nature present in the ES films has the main effect on the sensor performance, the response magnitude of the quartz electrode changes with the polarity characteristics strength concomitant with the actives sites number on the polymeric chain; it is worth noticeable that the polarity strength of ES films is derived from the dopant nature. Through all the conducted tests, it is concluded that the PANI-DBSA films has proven more efficiency than the two other films. On the other hand the film thickness has also played its role trough the proportional relationship between the $(\Delta f)$ magnitude and the thickness value. Increasing the film thicknesses enhances the chance for more electrostatic interactions and more active sites throughout the polymer chains.

\section{References}

[1] A.S. Bangalore, G.W. Small, R.J. Combs, R.B. Knapp, R.T. Kroutil, Automated detection of methanol vapour by open path Fourier transform infrared spectrometry, Anal. Chem. Acta 297, 387403 (1994); doi: 0003-2670 (94)00246-I.

[2] P.P. Tsai, I.C. Chen, M.H. Tzeng, Tin oxide $\left(\mathrm{SnO}_{x}\right)$ alcohol sensor from metal organic decomposed (MOD) thick film, Sensors and
Actuators B 13-14, 610-612 (1993); doi: 09254005/93/\$6.00.

[3] P.C. Chen, G. Shen, C. Zhou, Chemical sensors and electronic noses based on 1-D metal oxide nanostructures, IEEE Transactions on Nanotechnology, Vol. 7, No. 6, 668-682 (2008); doi: 10.1109/TNANO.2008.2006273.

[4] B. Adhikari, S. Majumdar, Polymers in sensor applications, Progress in Polymer Science 29, 699$766 \quad$ (2004); doi: $10 . \quad 1016 / \mathrm{j}$. progpolymsci.2004.03.002

[8] P. N. Bartlett, P. B. M. Archer, S. K. Ling-Chung, Conducting polymer gas sensors Part I: Fabrication and characterization, Sensors and Actuators B 19, 125-140 (1989); doi: 0250-6874/89/\$3.50.

[9] P. N. Bartlett, S. K. Ling-Chung, Conducting polymer gas sensors Part II: Response of polypyrrole to methanol vapour, Sensors and Actuators B 19, 141-150 (1989); doi: 0250$6874 / 89 / \$ 3.50$

[10] P. N. Bartlett, S. K. Ling-Chung, Conducting polymer gas sensors Part III: Results for four different polymers and five different vapours, Sensors and Actuators B 20, 287-292 (1989); doi: 0250-6874/89/\$3.50

[8] N.E. Agbor, M.C. Petty, A.P. Monkman, Polyaniline thin films for gas sensing, Sensors and Actuators B 28, 173-179 (1995); doi: 09254005/95/\$09.50 SSDI 0925-4005(95)01725-B.

[9] Z.F. Li, F.D. Blum, M.F. Bertino, C.S. Kim, S.K. Pillalamarri, One-step fabrication of a polyaniline nanofiber vapor sensor, Sensors and Actuators B 134, 31-35 (2008); doi:10.1016/j.snb.2008.04.009.

[10] L. Terlemezyan, P. Mokreva, D. Tsocheva, S. Peneva, K. Berovsky, T. Terov, Detection of free volumes in polyaniline complexes with various acids by using positron lifetime spectroscopy, Radiation Physics and Chemistry 77, 591-596 (2008); doi: 10.1016/j.radphyschem.2007.09.014 $\mu$. 